\title{
Molecular Adsorbent Recirculating System as a Diagnostic and Therapeutic Modality
}

\author{
Hicks SB $^{1}$ and Tabibian $\mathrm{JH}^{2,3 *}$
}

${ }^{1}$ Department of Internal Medicine, Mayo Clinic, Rochester, MN, USA

${ }^{2}$ Division of Gastroenterology and Hepatology, Mayo Clinic, Rochester, MN, USA

${ }^{3}$ Division of Gastroenterology, Olive View-UCLA Medical Center, Sylmar, CA, USA

"Corresponding author: Dr. James H. Tabibian, Division of Gastroenterology, Olive View-UCLA Medical Center, Sylmar, CA, 14445, USA, Tel: (747) 210-3205; Fax: (747) 210-4573; E-mail: jtabibian@dhs.lacounty.gov

Rec date: November 01, 2017; Acc date: November 23, 2017; Pub date: November 27, 2017

Copyright: (C) 2017 Hicks SB, et al. This is an open-access article distributed under the terms of the creative commons attribution license, which permits unrestricted use, distribution, and reproduction in any medium, provided the original author and source are credited.

\begin{abstract}
Despite the many advancements in liver transplantation (LT), mortality in patients with hepatic failure remains high, and to date, many patients die while awaiting LT. The molecular adsorbent recirculating system (MARS $B$ ) is an extracorporeal liver support system intended to provide short-term metabolic detoxification, often as a vital bridge to LT. We report the case of a 41-year-old non-Hispanic White male who developed worsening multi-factorial encephalopathy in the setting of decompensated alcoholic cirrhosis. He continued to deteriorate despite supportive medical therapy, and extensive investigation for alternative causes of encephalopathy aside from hepatic was unrevealing; as a result, there was concern that his encephalopathy was due to irreversible causes from which he may not recover appropriately following LT. We herein: i) describe the implementation of MARS as a diagnostic intervention for encephalopathy of uncertain etiology in a patient with end stage liver disease who, on the basis of prompt psychomotor improvement, underwent LT 19 days post-MARS implementation with an excellent clinical outcome and thus ii) propose the use of extracorporeal liver support not only as a short-term bridge but also as a diagnostic (and potentially therapeutic) measure in cases of cryptogenic encephalopathy, particularly in the setting of advanced liver disease.
\end{abstract}

Keywords: Hepatic encephalopathy; Cirrhosis; Prognosis; Liver transplantation; Bioartificial liver; Hemofiltration; Outcomes research

\section{Abbreviations: \\ MELD: Model End Stage Liver Disease; ALT: Alanine Aminotransferase; AST: Aspartate Aminotransferase; MARS: Molecular Adsorbent Recirculating System; MRI: Magnetic Resonance Imaging}

\section{Introduction}

Despite the many advancements in the field of liver transplantation (LT), mortality in patients who develop hepatic failure remains high, and many patients die while awaiting LT, in large part due to organ scarcity. To date, the most widely developed system for providing (short-term) metabolic detoxification and bridge to LT is the Molecular Adsorbent Recirculating System (MARS ${ }^{\circledR}$ ). MARS is a method of extracorporeal liver support based on the selective removal of albumin-bound toxins from the circulation which can facilitate simultaneous liver and kidney detoxification (Figure 1).

We present the case of a 41-year-old non-Hispanic White male who developed worsening encephalopathy in the setting of known decompensated alcoholic cirrhosis. He continued to deteriorate despite aggressive supportive medical therapy, thus prompting extensive investigation for alternative causes of encephalopathy. There was concern that a considerable proportion of his encephalopathy was due to irreversible causes such that he would not recover appropriately following LT. We herein report the implementation of MARS as a diagnostic and ultimately therapeutic intervention in in a patient with pre-LT endstage liver disease who was successfully bridged to LT and experienced excellent clinical recovery.

\section{Case Report}

A 41-year-old, non-Hispanic White male with a past medical history significant for decompensated alcoholic cirrhosis (model end stage liver disease (MELD) score of 30) presented to our institution for inpatient LT evaluation. Despite alcohol cessation eight months prior, his clinical condition continued to worsen, and he was requiring frequent hospitalizations for hepatic encephalopathy and acute on chronic kidney injury. His outpatient medications included lactulose, titrated to three bowel movements per day, rifaximin, zinc sulfate, furosemide, and spironolactone. On presentation, vital signs were unremarkable. Physical examination revealed a chronically illappearing, jaundiced man in no apparent acute distress. He exhibited hypokinetic dysarthria and was unable to respond appropriately to questioning in regard to orientation. $\mathrm{He}$ was bradyphrenic, bradykinetic, and unable to participate in examination due to features of encephalopathy. Asterixis was present. Motor examination revealed no focal deficits, and the patient was able to stand with some assistance. There was symmetric hyperreflexia without clonus throughout, and no response to plantar reflex. Laboratory evaluation yielded the following pertinent results (reference ranges provided in parentheses): hemoglobin $9.9 \mathrm{~g} / \mathrm{dL}$ (12.0-15.5 g/dL), leukocytes $12.9 \times$ $10^{9} / \mathrm{L}\left(3.5-10.5 \times 10^{9} / \mathrm{L}\right)$, platelets $98 \times 10^{9} / \mathrm{L}\left(150-450 \times 10^{9} / \mathrm{L}\right)$, sodium $140 \mathrm{mmol} / \mathrm{L}(135-145 \mathrm{mmol} / \mathrm{L})$, potassium $5.7 \mathrm{mmol} / \mathrm{L}$ (3.6-5.2 mmol/L), creatinine $1.8 \mathrm{mg} / \mathrm{dL}(0.8-1.3 \mathrm{mg} / \mathrm{dL})$, AST $98 \mathrm{U} / \mathrm{L}$ (8-48 U/L), ALT $58 \mathrm{U} / \mathrm{L}$ (7-55 U/L), alkaline phosphatase $168 \mathrm{U} / \mathrm{L}$ 


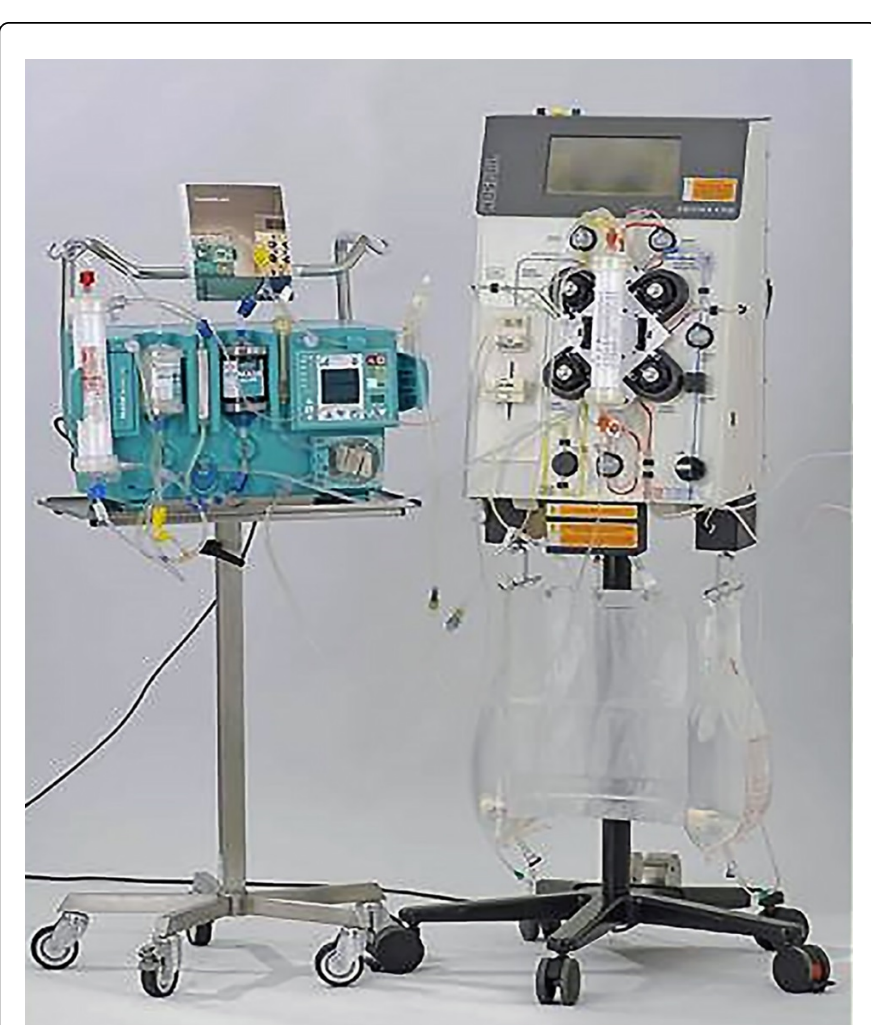

Figure 1: Molecular adsorbent recirculating (MARS) setup. On the left is the MARS unit, on the right the accompanying PRISMAFLEX continuous venovenous hemofiltration and therapeutic plasma exchange system (adapted with permission from Baxter, Deerfield, IL, USA).

(8-48 U/L), total bilirubin $18.8 \mathrm{mg} / \mathrm{dL} \quad(<1.2 \mathrm{mg} / \mathrm{dL})$, and international normalized ratio 1.9 . His model for end-stage liver disease (MELD-Na) score was 30. Despite appropriate treatment for hepatic encephalopathy, including lactulose, rifaximin, neomycin, zinc, and branched chain amino acids, the patient continued to exhibit the aforementioned mental status changes. Given the unclear etiology of his mental status changes, a concern was raised as to whether they would be reversible with LT. Resultantly, the patient's candidacy for LT was hindered and remained in question.

With the above considerations in mind, more extensive investigation was performed to delineate the etiology of the patient's persistent encephalopathy. A workup for reversible causes of dementia was negative. Magnetic resonance imaging of the brain showed bilateral, symmetric, patchy $\mathrm{T} 1$ hyperintensity in the basal ganglia region without evidence of neurodegenerative disease or other intracranial abnormality. Electroencephalogram showed nonspecific generalized slowing with predominant polymorphic delta activity compatible with severe diffuse metabolic encephalopathy. The Neurology inpatient service was consulted given the concern for potential nonreversible cause of cognitive impairment, and it was deemed he likely had multifactorial encephalopathy, though without further delineation as to whether it may be reversible (with LT). Additionally, the Psychiatry inpatient consultation service was called to examine the patient and to rule out catatonia-like illness (or other psychiatric disorder) as a potential cause for the patient's psychomotor retardation, the result of which was unrevealing.

One week into the patient's hospitalization, a multidisciplinary conference was held to decide on goals of care and the appropriateness of LT. There remained concern that a prohibitively large proportion of the patient's encephalopathy was due to irreversible causes such that he would not be a transplant candidate. Considering the patient's young age, the uncertainty surrounding his condition, and his otherwise favorable parameters for successful LT, it was suggested that MARS be implemented as a potentially diagnostic and therapeutic endeavor given he would otherwise likely expire short of LT. MARS was initiated the following day, and within three days, patient was more alert and mobile. By day five, the patient was answering questions sensibly, compared to previous minimal or no verbalization. Nineteen days after the initiation of MARS, the patient underwent uneventful LT and was discharged two weeks thereafter. Within approximately one year after LT, the patient returned to full-time work on his family farm and assisted with household activities. At his two-year follow-up, he continues to do well.

\section{Discussion}

MARS is a method of extracorporeal liver support where blood is dialyzed in a countercurrent fashion to an albumin containing solution. This process is implemented across a semipermeable membrane, which facilitates cleansing of the patient's blood of toxins. Importantly, there is a second circuit that utilizes activated charcoal and an anion exchanger, which serves to regenerate the albumin dialysate in preparation for further toxin removal (Figure 1) [1]. Extracorporeal liver support was initially developed by Strange et al. circa 1993 but has since undergone many improvements and variations (Figure 2) [2].

Since its inception, MARS has continued to establish a role as an effective short-term therapy in patients with end stage liver disease, including hepatic encephalopathy. It has been utilized primarily as a bridge to LT but has also served as a temporizing modality for those who have suffered acute liver failure. MARS has been shown to provide both protein- and albumin-bound toxin removal, including ammonium, biliary salts, urea, drug metabolites, and others, and in some cases of acute liver injury can help avoid the need for LT [3,4]. Also, it has been reported that MARS decreases serum concentrations of inflammatory mediators, which are considered to be a critical component of the neuropsychiatric findings associated with hepatic encephalopathy [5-7].

\section{Conclusion}

Moving forward, additional clinical trials are needed to more clearly characterize the role of MARS in the management of advanced liver disease as well as optimal patient selection. Similarly, and on the basis of the present case, it is plausible that MARS could have a new indication as a potential diagnostic as well as therapeutic maneuver in patients with medically refractory encephalopathy of uncertain etiology.

\section{Acknowledgement}

Grant support, conflicts of interest, writing assistance, or other disclosures: This work was supported in part by National Institutes of Health T32 training grant T32DK007198 (for J.H.T.) and National 
Citation: Hicks SB, Tabibian JH (2017) Molecular Adsorbent Recirculating System as a Diagnostic and Therapeutic Modality. J Mol Genet Med

Center for Advancing Translational Sciences grant UL1TR000135 (to the Mayo Center for Clinical and Translational Sciences, wherein J.H.T. was a PhD student).

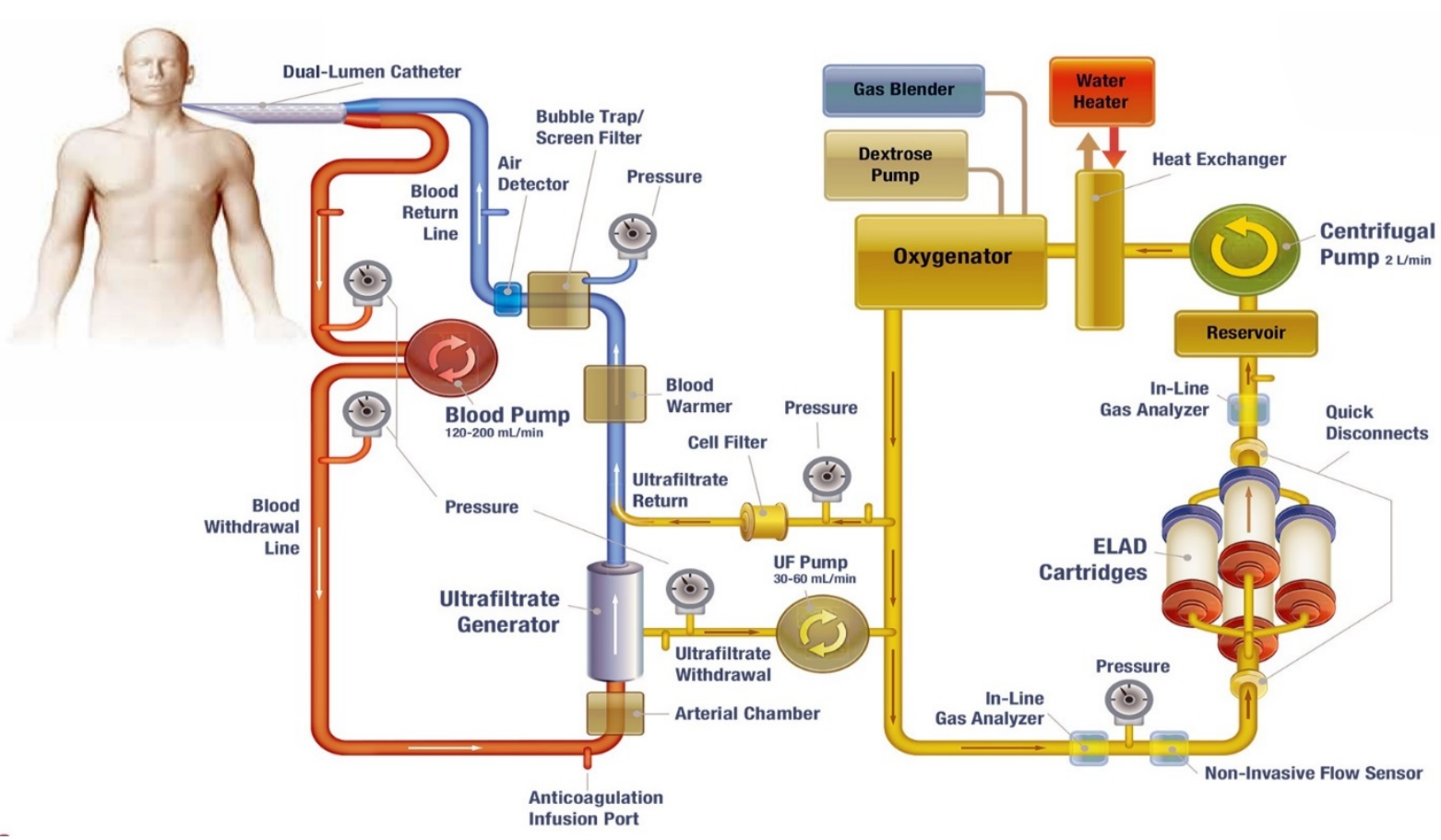

Figure 2: Human immortalized VTL C3A hepatocyte-based liver support system, ELAD (Vital Therapies, San Diego, CA, USA). Similar to MARS, this system is designed with the proposed intent to supplement hepatic function in order to improve survival rates among individuals with liver failure and is currently under investigation use.

\section{References}

1. Sen S, Williams R, Jalan R (2005) Emerging indications for albumin dialysis. Am J Gastroenterol 100: 468-475.

2. Stange J, Mitzner SR, Risler T, Erley CM, Lauchart W, et al. (1999) Molecular adsorbent recycling system (MARS): Clinical results of a new membrane-based blood purification system for bioartificial liver support. Artif Organs 23: 319-330.

3. Leise MD, Poterucha JJ, Kamath PS, Kim WR (2014) Management of hepatic encephalopathy in the hospital. Mayo Clin Proc 89: 241-253.

4. Diaz FC, Saez-Gonzalez E, Benlloch S, Alvarez-Sotomayor D, Conde I, et al. (2016) Albumin dialysis with MARS for the treatment of anabolic steroid-induced cholestasis. Ann Hepatol. 2016;15(6):939-943.
5. Dominik A, Stange J, Pfensig C, Borufka L, Weiss-Reining H, et al. (2014) Reduction of elevated cytokine levels in acute/acute-on-chronic liver failure using super-large pore albumin dialysis treatment: An in vitro study. Ther Apher Dial 18: 347-352.

6. Guo LM, Liu JY, Xu DZ, Li BS, Han H, et al. (2003) Application of molecular adsorbents recirculating system to remove $\mathrm{NO}$ and cytokines in severe liver failure patients with multiple organ dysfunction syndrome. Liver Int 23: 16-20.

7. Luo M, Guo JY, Cao WK (2015) Inflammation: A novel target of current therapies for hepatic encephalopathy in liver cirrhosis. World J Gastroenterol 21: 11815-11824. 